early identification and intervention for ADHD symptoms and emotional/behavior problems in children with a history of enterovirus $71 \mathrm{CNS}$ infection.

Increased risk of ADHD following CNS infection is not specific to enterovirus 71, but occurs with a variety of microorganisms. A recent review of etiologic factors, especially environmental causes, found an increased prevalence of ADHD in children born to women with a viral exanthematous rash of measles, varicella, or rubella during pregnancy. Other viral infections invoked include HIV, varicella zoster encephalitis, and influenza. (Millichap JG. Pediatrics 2008;121:e358-e365). A possible relation between ADHD and streptococcal infection, Borrelia burgdorferi and Lyme disease, or otitis media requires confirmation.

Clinical manifestations of enterovirus 71 infection are protean and include hand-footand mouth disease, brainstem encephalitis and polio-like paralysis. Isolation of the virus in cell culture is the standard diagnostic method, and stool and throat specimens produce the highest yield (AAP Redbook, $27^{\text {th }} \mathrm{ed}, 2006$ ). In a previous report of a long-term study of neurologic sequelae in 142 children with enterovirus $71 \mathrm{CNS}$ infection by the same group of investigators in Taiwan, children with cardiopulmonary complications had a significantly higher incidence of delayed neurodevelopment and lower IQ scores than children with CNS involvement alone. (Chang L-Y et al. N Engl J Med 2007;356:1226-1234).

ADHD is a highly heritable disorder, but various environmental factors, including viral infection may play a role in etiology. The recognition, prevention, and treatment of environmental causes may provide more effective management and reduce reliance on symptom modification with medication.

\title{
ROUTINE ECG AND STIMULANT MEDICATIONS FOR ADHD
}

The American Academy of Pediatrics (AAP) has issued a statement contradicting the recommendation of the American Heart Association (AHA) for routine electrocardiograms (ECGs) before starting medication to treat ADHD. The AAP and the American Academy of Child and Adolescent Psychiatry (AACAP) have concluded that sudden cardiac death (SCD) in persons taking medications for ADHD is a very rare event, occurring at rates no higher than in the general population. There is no evidence that routine ECG screening would prevent SCD. The AAP recommends careful assessment by a targeted cardiac history and examination, and a cardiac consultation only if clinically indicated. The AAP urges further research on risk factors for $\mathrm{SCD}$, and improved methods for detecting hidden cardiac disease. (Perrin JM, Friedman RA, Knilans TK, the Black Box Working Group and the Section on Cardiology and Cardiac Surgery. Pediatrics August 2008;122:451-453).

(Reprint orders: http://www.pediatrics.org/misc/reprints.shtml).

COMMENT. This AAP statement is endorsed by AACAP, Society for Developmental and Behavioral Pediatrics, and National Association of Pediatric Nurse Practitioners. The AAP recommends careful cardiac assessment of children with a history of cardiac disease, palpitations, syncope, or seizures; family history of sudden death; hypertrophic cardiomyopathy; long QT syndrome; or Wolff-Parkinson-White syndrome.

Risks of not treating ADHD include: 1) academic failure; 2) driving and other accidental injuries; 3) loss of self-esteem; 4) nicotine use and substance abuse; and 5) obesity. (see Ped Neur Briefs August 2008;22:64). 